\title{
Experimental induction of retrograde and anterograde amnesia concurrently: An animal model
}

\author{
RUSSELL E. MORGAN, ANGELA S. BURCH.VERNON, and DAVID C. RICCIO \\ Kent State University, Kent, Ohio
}

\begin{abstract}
In the clinical literature on memory dysfunction, the presence of both retrograde (RA) and anterograde (AA) amnesia due to acute trauma are commonly described. There has been, however, virtually no report of concurrently induced RA and AA using experimental procedures. In this study, we used two orthogonal tasks to examine the retrograde and anterograde impact of a single experimental insult. In Experiment 1, rats exposed to cold (hypothermia treatment) following consumption of a novel flavor, but prior to punishment training, showed impaired performance on later tests for attenuation of neophobia (retrograde task) and passive avoidance (anterograde task). The retrograde impairment was found to be time-dependent (Experiment 2) and independent of punishment effects (Experiment 3), indicating that both the retrograde and anterograde effects were based on memory loss. Therefore, it appears that RA and AA can be experimentally induced concurrently by a single traumatic insult. Thus, these findings provide an empirical model for comparing the characteristics of RA and AA when induced either independently or concurrently.
\end{abstract}

The presence of retrograde (RA) and anterograde amnesia (AA) following acute traumatic insult to the central nervous system is a common, albeit unfortunate, outcome described in the clinical literature on memory dysfunction. For example, in a study of over 1,000 patients with serious head injury, Russell and Nathan (1946) reported that over $90 \%$ suffered from both retrograde and anterograde memory impairments. In recent years, researchers have begun to investigate memory impairments of the transient, short-term type. Often referred to as transient global amnesia (TGA), patients with this type of short-term memory impairment usually exhibit both anterograde and retrograde amnesia (Kritchevsky \& Squire, 1989; Kritchevsky, Squire, \& Zouzounis, 1988). In most cases of TGA, the extent of RA is correlated with the severity of AA (Fisher \& Adams, 1964; Kritchevsky et al., 1988), both of which often improve within hours of onset. However, in more severe cases, such as closed-head injuries, memory recovery is often a much slower process that is sometimes never fully completed (Levin, Benton, \& Grossman, 1982; Schacter and Crovitz, 1977). Furthermore, although chronic insult may be qualitatively different from acute injury, many examples of AA accompanied by RA as a result of encephalitis, Korsakoff's syndrome, and other chronic disorders are common in the literature (Hirst, 1982).

The research reported in this paper was supported in part by NIMH Grant 37535 to D.C.R. We wish to recognize the valuable assistance of the late Mark Euken in conducting these experiments. Correspondence should be addressed to D. C. Riccio, Department of Psychology, Kent State University, Kent, $\mathrm{OH} \mathbf{4 4 2 4 2 .}$
Given the frequency with which both AA and RA are found as a consequence of a single acute incident, it is of interest that virtually none of the animal research on experimentally induced amnesia has examined the concurrent induction of these two phenomena. Thus, although there is an extensive body of research on RA resulting from traumatic insult (e.g., see McGaugh \& Herz, 1972; Riccio \& Richardson, 1984; Spear, 1978, for reviews) and a smaller but still substantial literature on AA (e.g., Bresnahan \& Routtenberg, 1980; Richardson, Riccio, \& Morilak, 1983), these findings are based on independent measurement of either RA or AA. Studies of each type of amnesia singly have provided important information on memory-principles, but they necessarily leave open the question of potential interactions or other complexities that might result when a single traumatic event gives rise to both forms of amnesia concurrently. Put somewhat differently, are the characteristics of AA and RA the same when they occur jointly as when they are obtained in isolation? Before such detailed comparisons can be made, however, it is necessary to demonstrate that concurrent induction and measurement of RA and AA can be achieved in a laboratory paradigm. Accordingly, establishing an animal analog that captures the central feature of the combination of amnesias seen in many clinical cases may prove instructive.

It should be noted that there is a quite reasonable explanation for the lack of research examining the concurrent production of AA and RA: Unlike the clinical situation, in which humans have experienced a multiplicity of relatively separate target episodes prior to, as well as after, the precipitating event, animal studies frequently use a single, well-defined learning event, typically involving 
rapid conditioning. This careful, analytic control over the learning episode is useful, but obviously precludes concurrent assessment of both amnesias. Introduction of a second task is potentially confounded by methodological problems related to task independence. For example, if shock-motivated passive- and active-avoidance tasks are used (before and after the traumatic insult), the linkage of common motivational states might mean that amnesia for one event could indirectly produce amnesia for the other task (see, e.g., Howard, Glendenning, \& Meyer, 1974; Howard \& Meyer, 1971; Robbins \& Meyer, 1970). Conversely, exposure to shock in the second task might tend to alleviate any amnesia for the initial task, just as noncontingent exposure to the unconditioned stimulus prior to testing has been shown to reduce or reverse the amnesia produced when RA and AA are induced separately (Meehan, Gordon, \& Riccio, 1988; Miller \& Springer, 1972).

Given these various considerations, the aim of the present study was to determine whether both types of amnesia might be obtained concurrently from a single precipitating event (hypothermia) in a paradigm employing two reasonably orthogonal learning tasks.

\section{EXPERIMENT 1}

In light of previous research from various labs, including our own, a passive-avoidance procedure was chosen as one of our two tasks. This passive-avoidance task appeared to be well suited for the concurrent paradigm, because it requires only one training trial and an abundance of research has indicated that it is sensitive to amnestic treatments.

The second task, which utilized attenuation of neophobia to a novel flavor, was based on work by Buresova and Bures (1980, 1981) and Richardson, Riccio, and Steele (1986). Buresova and Bures demonstrated that rats receiving deep-body cooling following initial consumption of a novel taste (apple juice) showed less attenuation of neophobia when the apple juice was presented a second time than did the controls, which did not receive body cooling. Thus, the amnestic (cooled) subjects responded to the apple juice as if it were still a novel substance; that is, their neophobia persisted. Richardson et al. demonstrated similar persistence of neophobia when rats were exposed to pentobarbital or shock-induced stress immediately after initial exposure to a novel taste. Thus, the attenuation of neophobia to a novel flavor appears to be sensitive to a number of amnestic treatments.

The attenuation-of-neophobia task met the criterion of rapid conditioning and susceptibility to hypothermiainduced amnesia; however, its use did place limitations on the design of the experiment. That is, exposure to the novel flavor always took place prior to the hypothermia treatment, whereas training of the passive-avoidance task always occurred after the hypothermia treatment. This asymmetrical design was necessary, because pilot data in- dicated that rats would not drink while in a cooled state (see also Buresova \& Bures, 1980).

\section{Method}

Subjects. Twenty-four male, naive Sprague-Dawley rats from the Holtzman Company were used. The subjects were 60-65 days old, and weighed 295-375 g (median weight $330 \mathrm{~g}$ ) at the start of the experiment. They were individually housed in wire-mesh cages, and were maintained on a 15:9-h light:dark cycle. Food was available ad lib throughout the experiment.

Apparatus. Unsweetened apple juice was presented to the subjects in calibrated drinking tubes. Throughout this and the following experiments, all apple juice exposures took place in the subjects' home cages. The passive-avoidance apparatus was a $43 \times 17 \times 17 \mathrm{~cm}$ Plexiglas chamber, divided into two equal compartments. One compartment, including the lid, was constructed of black Plexiglas; the other compartment was white and had a clear Plexiglas lid. A 15-W bulb was suspended $30 \mathrm{~cm}$ over the center of the white compartment. A guillotine doorway $(10 \times 8 \mathrm{~cm})$ connected the two compartments. The floor of the apparatus consisted of 2-mm stainless steel grids, spaced $1 \mathrm{~cm}$ apart. A matched impedance shock source was connected to the grids on the black side, to which a scrambled footshock ( $3 \mathrm{sec} / 0.7 \mathrm{~mA}$ ) could be delivered. Behavior for each subject was monitored visually by means of a tilted mirror placed under the center of the apparatus.

Procedure. The subjects were handled 4-5 min daily for 2 days prior to training. They were then randomly divided into three groups: Juice Only, No Hypo, and Hypo. Following handling on Day 2, all the subjects were water deprived. Twenty-four hours later, each subject was given a 4-min initial exposure to apple juice in its home cage. Just prior to this initial exposure, each subject had its food removed to ensure that apple juice flavor was not "masked" by eating during the brief exposure period. Also, each subject was handled for $1 \mathrm{~min}$ prior to initial and test exposure to the juice in order to assure that the animal was alert. After the 4-min juice exposure, the subjects in Group Juice Only simply had the drinking tube removed, and the amount consumed by each subject was recorded. Group Hypo also had the drinking tube removed after $4 \mathrm{~min}$; however, they were then exposed to deep-body cooling (hypothermia). Hypothermia was induced by immersing the rat, via a restraining tube, up to the neck in $2^{\circ}-3^{\circ} \mathrm{C}$ water until its colonic temperature, as measured by a Fisher Scientific digital thermometer, dropped to $21^{\circ} \pm 1^{\circ} \mathrm{C}$. Fear conditioning took place once the hypothermic rats rewarmed to $30^{\circ} \pm 1^{\circ} \mathrm{C}$ (mean interval to rewarm was $80 \mathrm{~min}$ ). In contrast, Group No Hypo, after removal of the drinking tube, was simply taken to the "rewarming room" and fear conditioned after an 80-min delay in order to match the rewarming time of Group Hypo.

The fear-conditioning procedure began with placement of the rat in the white side of the passive-avoidance apparatus, facing away from the guillotine door. After $10 \mathrm{sec}$, the door was lifted. Once the rat crossed over to the black compartment (all four paws), the door was shut and a 3-sec unscrambled footshock was delivered. After fear conditioning, which was the same for Groups Hypo and No Hypo, all the subjects were returned to the colony room where food and water were made available $1-2 \mathrm{~h}$ later. Throughout this and the following experiments, the experimenter measuring juice consumption was "blind" to the subject's group designation.

Water bottles were again removed $24 \mathrm{~h}$ after the initial juice exposure. All the groups were tested after this second $24-h$ waterdeprivation period. For Group Juice Only, testing simply consisted of a second 4-min exposure to the apple juice. However, Groups Hypo and No Hypo were given both a second exposure to the juice and a 10-min passive-avoidance test. This passive-avoidance test consisted of placing the rat on the white (safe) side of the training apparatus, facing away from the door. After $10 \mathrm{sec}$, the door was 
removed. Initial latency to cross into the black side and total time spent in the white compartment (TTW) were the measures recorded. The order of the two tests was counterbalanced for both groups; however, no effect of order was found. Therefore, the data were combined for statistical analyses.

\section{Results and Discussion}

To assess the retention of information about initial exposure to apple juice, difference scores were calculated by subtracting the amount consumed at initial presentation from the amount consumed at test presentation. These data are depicted in Figure 1. A Kruskal-Wallis analysis of variance (ANOVA) on the difference scores revealed a significant treatment effect $[H(2)=12.66, p<.002]$. Subsequent Mann-Whitney $U$ tests revealed that the two noncooled groups (Juice Only and No Hypo) did not differ from each other $(U=31.0, p=.31)$; however, both noncooled groups did differ from Group Hypo $(U=4.5$, $p<.01 ; U=18.5, p<.05$ ). The finding that Groups Juice Only and No Hypo did not differ indicates that the fear-conditioning task (passive avoidance) did not interfere with the attenuation of neophobia. The persistence of neophobia in Group Hypo, however, suggests that the cooled subjects failed to remember the initial exposure to the novel flavor-an outcome interpretable as retrograde amnesia.

Data from the passive-avoidance task were analyzed in terms of both the latency and TTW data. Because both measures revealed the same pattern of results, only the latency data are presented here. As Figure 2 suggests, initial crossover latencies for the two groups trained in the passive-avoidance task (Hypo and No Hypo) did not differ $(H=0.34, p>.05)$, but Group Hypo displayed shorter latency scores than the noncooled (No Hypo) subjects at testing $(H=7.14, p<.008)$. Thus, deep-body cooling prior to passive-avoidance training resulted in an anterograde impairment for the fear-conditioning experience.

The data from this first experiment apparently indicate that when tested at normal body temperature, the previously cooled subjects displayed impaired retention per-

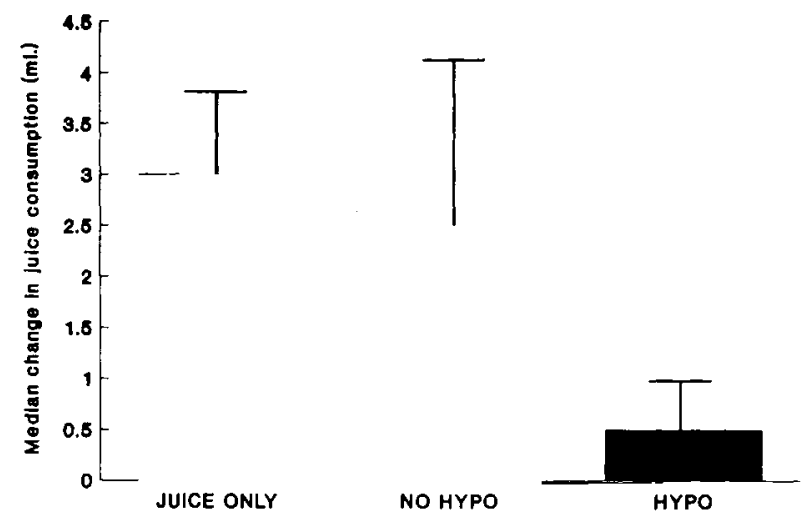

Figure 1. Median change in juice consumption from training exposure to test exposure across experimental conditions. Brackets indicate semi-interquartile ranges.

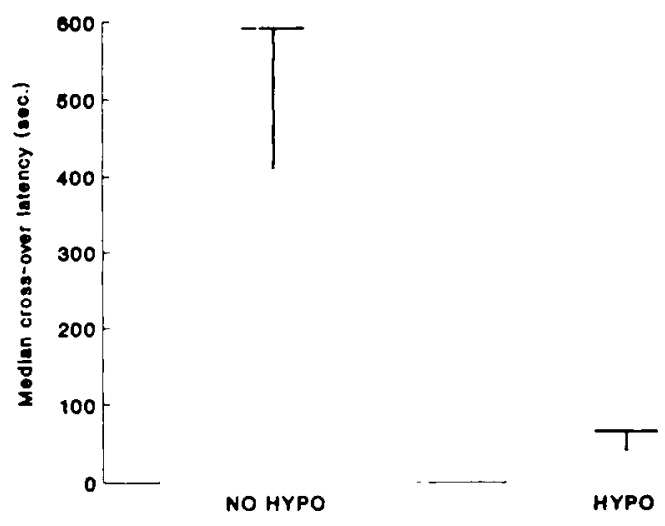

Figure 2. Median passive-avoidance test latencies obtained for rats given hypothermic treatment (Hypo) or no hypothermic treatment (No Hypo). Brackets indicate semi-interquartile ranges.

formance on both the attenuation of neophobia (retrograde) and passive-avoidance (anterograde) tasks. However, because passive-avoidance conditioning was conducted while the rats were hypothermic, it remained possible that the observed anterograde impairment was the result of acquisition failure, rather than memory impairment. To address this concern, a subsequent group $(n=10)$ was run, which underwent training procedures identical to Group Hypo; however, these rats were tested immediately after training (while still hypothermic) and again $24 \mathrm{~h}$ later. When tested immediately after training, these 10 rats demonstrated ceiling-level retention scores (median = $600 \mathrm{sec}$ ), as opposed to the short crossover latencies shown both before training (median $=6 \mathrm{sec}$ ) and $24 \mathrm{~h}$ later (median $=38 \mathrm{sec}$ ). This finding suggests that the anterograde impairment present at testing was memory based, rather than an acquisition deficit. Furthermore, previous studies from this laboratory have indicated that learning in the anterograde hypothermic state is not quantitatively or qualitatively different from that occurring under "normal" conditions (Richardson et al., 1983; Santucci, Kasenow, Riccio, \& Richardson, 1987).

\section{EXPERIMENT 2}

Although the findings from Experiment 1 clearly demonstrated induction of concurrent retrograde and anterograde performance deficits, possible alternative interpretations of the retrograde impairment (i.e., punishment-based effects) must be addressed before these deficits can be attributed to memory impairments.

As mentioned earlier, Buresova and Bures $(1980,1981)$ initially found that deep-body cooling was capable of disrupting attenuation of neophobia. However, to the best of our knowledge, this procedure has not been systematically investigated outside of Buresova and Bures's work. Therefore, it was important to investigate whether the retrograde effects of hypothermia on this task would follow the usual time-dependent characteristics of RA. That 
is, would delays between training (initial juice consumption) and cooling result in temporally graded severity of amnesia?

\section{Method}

Subjects. Twenty-one male, naive Sprague-Dawley rats from the Holtzman Company served as subjects. They were housed identically to those in Experiment 1.

Apparatus. The apparatus was the same as that used in Experiment 1.

Procedure. All handling, conditioning, and testing procedures were identical to those previously employed, except as stated below.

Three groups of rats were given a 4-min initial exposure to apple juice after $24 \mathrm{~h}$ of water deprivation. The subjects were then exposed to deep-body cooling either immediately (No Delay), $10 \mathrm{~min}$ (10-Min Delay), or $60 \mathrm{~min}$ (60-Min Delay) after juice exposure. All three groups were then trained in the passive-avoidance task once they returned to $30^{\circ} \pm 1^{\circ} \mathrm{C}$. In this design, Group No Delay served as a replication of Group Hypo from Experiment 1.

Forty-eight hours after juice exposure, and after a second 24-h water-deprivation period, all the subjects were tested on both the attenuation of neophobia and passive-avoidance tasks. As in Experiment 1, changes in juice consumption, test latency, and TTW data were recorded. In addition, order of testing was again counterbalanced.

\section{Results and Discussion}

The findings of major relevance concern the change in juice consumption from first to second exposure as a function of delay between initial juice exposure and hypothermic treatment. As in Experiment 1, in order to increase the sensitivity of our measure and to take into account individual differences in consumption, difference scores were calculated by subtracting the amount consumed at initial presentation from the amount consumed at test presentation. These data are presented in Figure 3. A KruskalWallis ANOVA on the difference scores revealed a significant treatment effect $(H=7.29, p<.03)$. Subsequent Mann-Whitney $U$ tests indicated that Group 60-Min Delay differed from Groups No Delay and 10-Min Delay ( $U=$ $7.0, p<.02 ; U=5.5, p<.04$ ). However, Groups No Delay and 10-Min Delay did not differ from each other $(U=19.5, p>.05)$.

These results indicate that if the hypothermia treatment was initiated within 10 min after initial juice exposure, retrograde performance deficits were produced. However, if initiation of the hypothermic treatment was delayed for $60 \mathrm{~min}$, little evidence for retrograde impairment was obtained. Thus, attenuation of neophobia varied as an inverse function of training-to-treatment delay-an outcome consistent with previous findings on retrograde amnesia.

In Experiment 2, the anterograde task (passive-avoidance training) was included in order to replicate the design of Experiment 1. Because all three groups in the current experiment were fear conditioned at the same body temperature, the data from the passive-avoidance test was expected to indicate equally severe memory impairment for all three groups. As expected, data from all three groups were comparable. Median test latencies for Groups No Delay, 10-Min Delay, and 60-Min Delay were $27 \mathrm{sec}$,

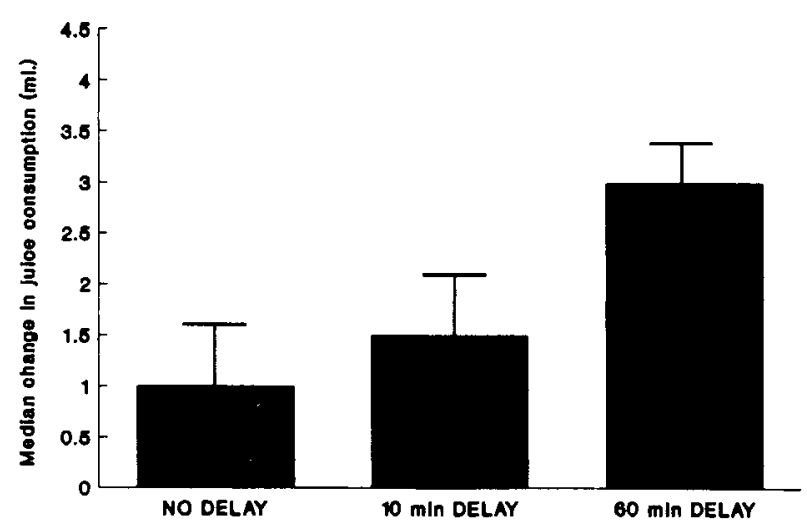

Figure 3. Median change in juice consumption for subjects experiencing delays of 0,10 , or $60 \mathrm{~min}$ between initial juice exposure and cooling. Brackets indicate semi-interquartile ranges.

$12 \mathrm{sec}$, and $21 \mathrm{sec}$, respectively, and thus did not differ $(H=1.01, p>.05)$.

\section{EXPERIMENT 3}

Experiments 1 and 2 provided evidence that a single, acute insult (hypothermia) can result in both retrograde and anterograde performance deficits, and that the retrograde impairment is time dependent. However, the possibility remained that the hypothermic treatment following initial juice exposure may have had a punishment effect, which would also be expected to diminish as a function of the delay interval. That is, because the stimuli associated with initial juice exposure occurred in close temporal proximity to the hypothermia treatment, they could acquire aversive properties. If so, this conditioned aversion could serve as an alternative explanation for the lack of attenuation of neophobia at the short intervals. However, a number of studies argue against a punishment interpretation. Riccio, Gaebelein, and Cohen (1968) demonstrated through the use of pseudotrained (nonshocked) controls that the retrograde amnesic effects of hypothermia on performance of a passive-avoidance task were not due to punishment. Of greater relevance to the present question is the finding that hypothermia apparently cannot serve as an unconditioned stimulus in conditioned tasteaversion experiments (Haroutunian \& Riccio, 1975; Ionescu \& Buresova, 1977; Matthews, MacArdy, \& Riccio, 1989). In these experiments, saccharin consumption was followed by deep-body cooling. In each instance, the investigators found no evidence that saccharin preference was modified by the cooling treatment.

Although these previous findings make a punishment interpretation highly unlikely, an investigation of this possibility under the conditions employed in the present experiments seemed desirable. Thus, in Experiment 3, we attempted to determine directly whether punishment effects were involved in the failure to attenuate neophobia in cooled subjects. 
The design for Experiment 3 was based on a study by Wetsel, Riccio, and Hinderliter (1976), which showed that the retrograde amnesic effects of hypothermia, in a passive-avoidance paradigm, could be eliminated if rats were artificially rewarmed soon after cooling. Wetsel et al. suggested that a "release" from the retrograde influences of hypothermia may have occurred because of the rapid return to normal body temperature, providing an opportunity for memory to become associated with "normal" as well as cold-state cues. Thus, in a manner similar to state-dependent views of RA, rapid rewarming may weaken RA by providing some overlap between contextual cues present at acquisition and those at testing.

In the present study, rats received the same initial juice exposure and cooling treatment as in Experiment 1, and then were rapidly rewarmed. If the failure to attenuate neophobia in Experiments 1 and 2 was due to the amnestic effects of the hypothermia treatment, then the rewarming manipulation should result in attenuation of neophobia due to its ability to prevent RA. However, if the failure to attenuate neophobia in Experiments 1 and 2 was punishment based, then the rewarming treatment would not be expected to fully attenuate the neophobia. The latter conclusion is based on the fact that the rewarmed rats are subjected to the same immediate cold exposure and hypothermia induction as rats from Experiment 1, and thus the possible punishment contingency (train/cool) remains in place.

\section{Method}

Subjects. Thirty-nine male, naive Sprague-Dawley rats from the Holtzman Company served as subjects. They were housed in the same manner as those in the previous two experiments.

Apparatus. The apparatus was the same as that used in the previous experiments.

Procedure. After 2 days of handling and $24 \mathrm{~h}$ of water deprivation (as in Experiments 1 and 2), the subjects were randomly divided into three groups of 13 per group. All the subjects were then given a 4-min initial apple juice exposure, followed by either immediate cooling (Group Hypo), immediate immersion for $20 \mathrm{~min}$ in warm $\left(36.4^{\circ} \mathrm{C}\right.$ ) water (Group Warm), or immediate cooling followed by a 20 -min immersion in warm $\left(36.4^{\circ} \mathrm{C}\right.$ ) water (Group Hypo/Warm). Twenty minutes of immersion in $36.4^{\circ} \mathrm{C}$ water was chosen because previous work from our lab has indicated that this procedure is sufficient to return hypothermic rats to within $1^{\circ}$ of normal body temperature, without resulting in hyperthermic-induced amnesia (Wetsel et al., 1976). Group Hypo was trained in the passive-avoidance task once they returned to $30^{\circ} \pm 1^{\circ} \mathrm{C}$ under room-temperature conditions. Previous work in our lab has indicated that rats that are cooled to $21^{\circ} \pm 1^{\circ} \mathrm{C}$ require a mean time of $80 \mathrm{~min}$ to return to $30^{\circ} \pm 1^{\circ} \mathrm{C}$ under room-temperature conditions. Thus, in order to keep the delay between immersion and passive-avoidance training equal for all the groups, Groups Warm and Hypo/Warm were trained in the passiveavoidance task 80 min after completion of their immersion period.

Once again, testing was conducted $48 \mathrm{~h}$ later, after a second $24-\mathrm{h}$ water-deprivation period. As in Experiments 1 and 2, change in juice consumption, test latency, and TTW data were recorded. However, in this case, order of testing was not counterbalanced; rather, second-exposure juice consumption was always tested first. We did not counterbalance the order of testing, because (1) Experiments 1 and 2 indicated that order of testing did not have a significant effect, and (2) we wanted to maximize the sensitivity of the test in detecting any punishment effects, as reflected in continued neophobia to the juice.

\section{Results and Discussion}

Difference scores were again calculated by subtracting the amount consumed at initial presentation from the amount consumed at test presentation. A Kruskal-Wallis ANOVA on these difference scores (presented in Figure 4) revealed a significant treatment effect $(H=9.38, p<$ .009). Subsequent Mann-Whitney $U$ tests revealed that Group Hypo/Warm increased their consumption of apple juice more than Group Hypo, at a marginal level of significance $(U=48.5, p<.06)$ (see Figure 4$)$. As expected, Groups Hypo and Warm differed significantly $(U=30.0, p<.004)$, and Groups Hypo/Warm and Warm did not differ $(U=54.0, p>.10)$. Taken together, the pattern of results indicates that artificial rewarming immediately after the cooling treatment greatly reduces impairment on the neophobia task. Although the subjects in Group Hypo/Warm were cooled to the same level as Group Hypo, the subsequent rewarming manipulation released them from the retrograde effects of the cooling treatment. Thus, able to recall their initial exposure to the apple juice, the Hypo/Warm subjects consumed more juice at the second exposure.

As in Experiment 2, the data from the passive-avoidance task were of secondary importance to the aim of Experiment 3. However, the data are worthy of consideration because they offer a third opportunity to replicate the concurrent impairments first found in Experiment 1. Recall that in this experiment, Group Hypo was the only group to be trained in the passive-avoidance task while remaining in a cooled $\left(30^{\circ} \pm 1^{\circ} \mathrm{C}\right)$ state. Groups Hypo/Warm and Warm received fear conditioning at essentially normal body temperature. As expected, test data (depicted in Figure 5) from Group Hypo indicated a high level of impairment on the passive-avoidance task compared with Group Warm $(U=11.0, p<.001)$. One unexpected finding was that Group Hypo/Warm differed from Groups Warm and Hypo $(U=29.0, p<.004 ; U=48.5, p<.06)$.

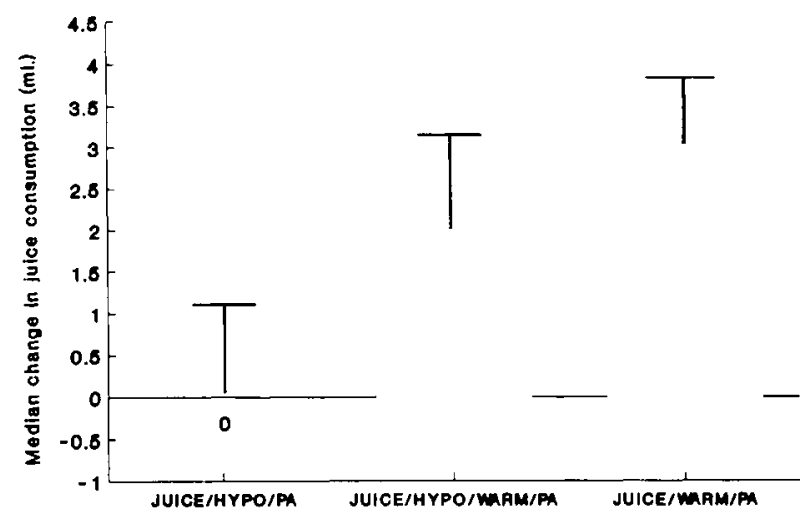

Figure 4. Median change in juice consumption exhibited by subjects that were cooled only (Hypo); cooled, then warmed (Hypo/Warm); or warmed only (Warm). Brackets indicate semi-interquartile ranges. 


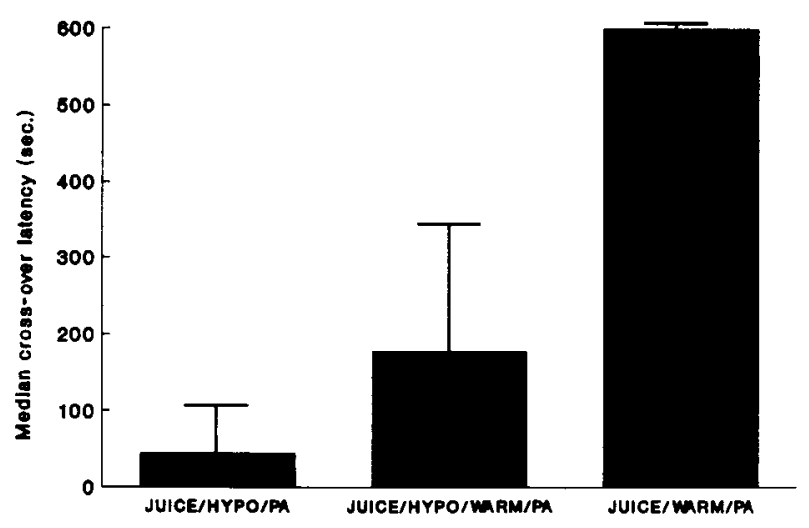

Figure 5. Median passive-avoidance test latencies obtained for rats that were cooled only (Hypo); cooled, then warmed (Hypo/Warm); or warmed only (Warm). Brackets indicate semi-interquartile ranges.

Thus, although Group Hypo/Warm had returned to normal body temperature at the time of passive-avoidance training, they still showed an intermediate degree of anterograde impairment. Although this peculiar finding was obtained in two replications of Experiment 3, later work undertaken to further explore this potentially interesting effect was unable to confirm the finding (i.e., the anterograde impairment was no longer attainable).

\section{GENERAL DISCUSSION}

The present series of experiments clearly demonstrates that a single hypothermia treatment can result in both AA and RA concurrently. In each of the three experiments, induction of hypothermia before passive-avoidance training and after initial juice exposure resulted in impaired performance on both tasks when subjects were later tested at normal body temperature. In Experiments 2 and 3, we investigated whether hypothermia's retrograde effects on the neophobia task were similar to its known effects on aversively motivated tasks. Indeed, the retrograde impairment resulting from deep-body cooling after initial juice exposure followed the usual time-dependent relationship between training and initiation of the amnestic treatment; that is, increased training-treatment delay resulted in reduced memory impairment. Immediate rewarming after induction of hypothermia (Experiment 3 ) also resulted in attenuation of amnesia, as has been shown to occur with aversively motivated tasks (Wetsel et al., 1976).

As previously discussed, Experiment 3 was also an attempt to rule out a punishment interpretation of hypothermia's retrograde effects in the present paradigm. The subjects in this third experiment were artificially rewarmed immediately after having undergone cooling. Although these rewarmed rats were subjected to the same initial cooling procedure that resulted in amnesia in nonrewarmed animals, they did not display the persistence of neophobia that characterized the RA effects. Had the retrograde impairment in cooled rats been due to punish- ment effects, one would expect a similar impairment in the rewarming condition, because rewarming did not interrupt the temporal contiguity of the initial juice exposure and subsequent cooling treatment. Furthermore, as previously mentioned, a punishment interpretation of the retrograde effects seen in this paradigm is also highly unlikely because there is evidence that hypothermia apparently cannot serve as an unconditioned stimulus in conditioned taste-aversion experiments (Haroutunian \& Riccio, 1975; Ionescu \& Buresova, 1977; Matthews et al., 1989).

We have suggested that the frequent coexistence of RA and $\mathrm{AA}$ as a consequence of naturally occurring acute insults in humans deserves attention in animal research. The present data encourage the view that it is possible to develop an animal model incorporating some of the salient features of such memory dysfunctions. We would acknowledge, however, that the study of memory phenomena does not demand such a "naturalistic" preparation. In the determination of principles governing encoding, storage, and retrieval, there is no compelling reason that experiments with amnesia (or any other phenomenon) should attempt to parallel the multiple aspects of "reallife" phenomena; the usefulness of the analytic, reductionistic approach has been amply demonstrated.

However, we also believe that the present paradigm may prove heuristically useful in at least two respects. First, it will now be possible to compare the characteristics of AA and RA when induced singly or concurrently. This is the issue of interactive effects of the two amnesias. For example, is the slope of the temporal gradient of RA the same in both instances? Second, and of perhaps greater theoretical significance, is the investigation of issues related to the alleviation of amnesia. Although there is good reason to believe that reinstatement of the hypothermia state is potentially capable of reversing both AA and RA (Richardson, Guanowsky, Ahlers, \& Riccio, 1984; Hinderliter, Webster, \& Riccio, 1975), it will be of interest to determine the efficacy of other reminder treatments. Exposure to noncontingent footshock has been widely used to alleviate amnesia for tasks based on footshock as the unconditioned stimulus. But will such a manipulation also lead to recovery of the memory for the orthogonal task, or will it be specific to the footshock task? If memory for both tasks is "linked" through association with the amnestic treatment (see, e.g., Hinderliter et al.), then alleviation of AA as well as RA is an interesting possibility.

\section{REFERENCES}

Bresnahan, E. L., \& Routtenberg, A. (1980). Medial forebrain bundle stimulation during learning and subsequent retention disruption. Physiological Psychology, 8, 112-119.

Buresova, O., Bures, J. (1980). Post-ingestion interference with brain function prevents attenuation of neophobia in rats. Behavioral Brain Research, 7, 299-312.

Buresova, O., \&uRES, J. (1981). Threshold hypothermia disrupting acquisition of conditioned taste aversion and attenuation of neophobia in rats. Behavioral \& Neural Biology, 31, 274-282.

Fisher, C. M., \& ADAMs, R. D. (1964). Transient global amnesia. Acta Neurologica Scandinavia, 40, 1-83. 
Haroutunian, V., \&iccio, D. C. (1975). Acquisition of rotationinduced taste aversion as a function of drinking-treatment delay. Physiological Psychology, 3, 273-277.

Hinderliter, C. F., Webster, T., \& Riccio, D. C. (1975). Amnesia induced by hypothermia as a function of treatment-test interval and recooling in rats. Animal Learning \& Behavior, 3, 257-263.

HiRst, W. (1982). The amnestic syndrome: Descriptions and explanations. Psychological Bulletin, 91, 435-460.

Howard, R. L., Glendenning, R. L., \& Meyer, D. R. (1974). Motivational control of retrograde amnesia: Further explorations and effects. Journal of Comparative \& Physiological Psychology, 86, 187-192.

HowARD, R. L., MEyer, D. R. (1971). Motivational control of retrograde amnesia in rats: A replication and extension. Journal of Comparative \& Physiological Psychology, 74, 37-40.

IONESCU, E., \& BuREsova, O. (1977). Effects of hypothermia on the acquisition of conditioned taste aversion in rats. Journal of Comparative \& Physiological Psychology, 91, 1297-1307.

KRITChEVSKY, M., SQUIRE, L. R. (1989). Transient global amnesia: Evidence for extensive, temporally graded retrograde amnesia. Neurology, 39, 213-218.

KritcheVSKY, M., SQuire, L. R., Z Zouzounis, J. A. (1988). Transient global amnesia: Characterization of anterograde and retrograde amnesia. Neurology, 38, 213-219.

Levin, H. S., Benton, A. L., \& Grossman, R. G. (1982). Neurobehavioral consequences of closed head injury. Oxford: Oxford University Press.

Matthews, K., MacArdy, E. A., \& Riccio, D. C. (1989, May). Thermoregulatory disruption in rats fails to produce conditioned taste aversion. Paper presented at the 61st Annual Meeting of the Midwestern Psychological Association, Chicago, IL.

McGaugh, J. L., \&erz, M. J. (1972). Memory consolidation. San Francisco: Albion.

Meehan, S. M., Gordon, T., \& Riccio, D. C. (1988, May). Differential effectiveness of footshock in attenuating the memory loss produced by hypothermia-induced anterograde amnesia and pentobarbital state dependent retention. Paper presented at the meeting of the Midwestern Psychological Association, Chicago, IL

Miller, R. R., \& SPRINGeR, A. D. (1972). Induced recovery of memory in rats following electroconvulsive shock. Physiology \& Behavior, 8, 645-651.
Riccio, D. C., Gaenelein, C., Cohen, P. (1968). Some behavioral aspects of retrograde amnesia produced by hypothermia. Physiology \& Behavior, 3, 973-976.

Riccio, D. C., \& Richardson, R. (1984). The status of memory following experimentally induced amnesias: Gone, but not forgotten. Physiological Psychology, 12, 59-72.

Richardson, R., Guanowsky, V., Ahlers, S. T. . Riccio, D. C (1984). Role of body temperature in the onset of, and recovery from, hypothermia-induced anterograde amnesia. Physiological Psychology, 12, 125-132.

Richardson, R., Riccio, D. C. . Morilak, D. (1983). Anterograde memory loss induced by hypothermia in rats. Behavioral \& Neural Biology, 37, 76-88.

Richardson, R., Riccio, D. C., Steele, J. H. (1986). Statedependent retention induced by postacquisition exposure to pentobarbital or shock stress in rats. Animal Learning \& Behavior, 14, 73-79.

Robins, M. J., \& MEYER, D. (1970). Motivational control of retrograde amnesia. Journal of Experimental Psychology, 84, 220-225.

Russell, W. R., \& NAthAN, P. W. (1946). Traumatic amnesia. Brain, 69, 280-300.

Santucci, A. C., Kasenow, P. M., Riccio, D. C., \& Richardoson, R. (1987). Hypothermia-induced anterograde amnesia: Is memory loss attributable to impaired acquisition? Behavioral \& Neural Biology. 48, 13-23.

SChacter, D. L., Crovitz, H. F. (1977). Memory function after closed head injury: A review of the quantitative research. Contex, 13. 150-176.

SPEAR, N. E. (1978). The processing of memories: Forgetting and retention. Hillsdale, NJ: Erlbaum.

Wetsel, W. C., Riccio, D. C., Hinderliter, C. F. (1976). Effects of artificial rewarming upon hypothermia-induced retrograde amnesia. Physiological Psychology, 4, 201-206.

(Manuscript received October 28, 1992 ; revision accepted for publication April 8, 1993.) 